\title{
Some data on the distance-neighbour function for relative diffusion
}

\author{
By PAUL J. SULLIVAN† \\ W.M. Keck Laboratory, California Institute of Technology, Pasadena, California
}

(Received 3 October 1969 and in revised form 9 January 1971)

Repeated observations of dye plumes on Lake Huron are interpreted according to the theoretical proposals of Richardson (1926) and Batchelor (1952) about the characteristics of a dispersing eloud of marked fluid within a field of homogeneous turbulence. The results show the average of several instantaneous concentration distributions about their centre of gravity to be approximately Gaussian and the distance-neighbour function to be of approximately Gaussian form. The data are consistent with the theoretical description given by Batchelor, namely,

$$
q(y, t)=\left(2 \pi \overline{y^{2}}\right)^{-\frac{1}{2}} \exp \left(-y^{2} / 2 \overline{y^{2}}\right), \quad\left(\overline{y^{2}}=\left(\frac{2}{3} \alpha t\right)^{3}\right),
$$

where $q(y, t)$ is the distance-neighbour function and $\alpha$ is the constant of the

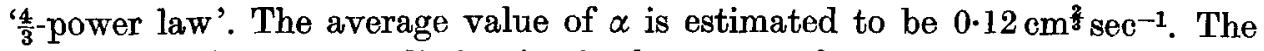
rate of turbulent energy dissipation in the near-surface currents of Lake Huron is estimated as $\epsilon \sim 2 \cdot 1 \times 10^{-3} \mathrm{~cm}^{2} \mathrm{sec}^{-3}$.

\section{Introduction}

The characteristics of a dispersing cloud of marked fluid in a field of homogeneous turbulence have been considered by Richardson $(1926,1929)$ and Batchelor (1952). Richardson (1926) pointed out that relative dispersion is an aocelerating process in which an initially marked volume of fluid is spread at a rate dependent upon its size. Richardson summarized various atmospheric diffusion data (over the range of $1 \mathrm{~m}$ to $10 \mathrm{~km}$ ) and arrived at the $\frac{4}{3}$-power law' for the relative diffusion coefficient $K$ defined by

$$
K=\alpha y^{\frac{4}{3}}
$$

where $y$ is the distance separating two typical marked fluid elements and $\alpha$ is a constant. Richardson \& Stommel (1948) and Ozmidov (1957, 1960) found that (1) gives a good description of the relative diffusion of floating objects in the range $2 \mathrm{~m}$ to $2 \mathrm{~km}$. A theoretical interpretation of relation (1) has been given by Batchelor $(1950,1952)$ in terms of the universal similarity theory of Kolmogoroff. Thus for the inertial subrange of high Reynolds number flow, Batchelor deduced that

$$
K=c \epsilon^{\frac{1}{3}} y^{\frac{4}{3}},
$$

$\dagger$ Permanent address: Department of Applied Mathematics, The University of Western Ontario, London 72, Ontario, Canada. 
where $c$ is a constant of order unity and $\epsilon$ is the rate of energy dissipation. The $\frac{4}{3}$ law was, however, sometimes found when the turbulence was not isotropic and the applicability of (2) to objects floating on the sea surface is not elear.

To describe the shape characteristics of a spreading cloud, Richardson (1926) introduced the distance-neighbour function $q(y, t)$ defined by

$$
q(y, t)=\frac{1}{A} \int_{-\infty}^{\infty} C(x+y, t) C(x, t) d x,
$$

where

$$
A=\int_{-\infty}^{\infty} C(x, t) d x \text { and } C(x, t)
$$

is the instantaneous concentration distribution in the dimension $x$ at the time $t$. $q(y, t)$ is an even function and its second and fourth moments are related to those of the concentration curve by

$$
\begin{aligned}
\sigma^{2} & =\frac{1}{2} \overline{y^{2}}, \\
\mu & =\frac{1}{2} \overline{y^{4}}-\frac{3}{4}\left(\overline{y^{2}}\right)^{2},
\end{aligned}
$$

where $\sigma^{2}$ and $\mu$ are the second and fourth moment of $C(x)$ about its centre of gravity at a given time $t$.

Richardson suggested the differential equation

$$
\frac{\partial q}{\partial t}=\frac{\partial}{\partial y}\left(\alpha y^{\frac{4}{3}} \frac{\partial q}{\partial y}\right)
$$

to describe the variable $q$. This has a solution

$$
q(y, t)=M\left(2 / 3 \pi^{\frac{1}{2}}\right)(9 / 4 \alpha t)^{\frac{3}{2}} \exp \left(-9 y^{\frac{2}{3}} / 4 \alpha t\right), \quad \overline{y^{2}}=\frac{35}{8}\left(\frac{2}{3} \alpha t\right)^{3}
$$

for the initial condition

$$
q(y, 0)=M \delta(y)
$$

together with the constraint $\int_{-\infty}^{\infty} q(y, t) d y=M$.

$\delta(y)$ is the Dirac delta function.

The Richardson formulation implies that the spreading of two marked fluid elements depends upon their instantaneous random separation. An alternative equation, in which the effective diffusivity depends upon the statistical quantity $\overline{y^{2}}$, was given by Batchelor (1952) as

$$
\partial q / \partial t=\alpha\left(\overline{y^{2}}\right)^{\frac{2}{3}} \partial^{2} q / \partial y^{2}
$$

The solution satisfying the above conditions is here

$$
\left.q(y, t)=\left(2 \pi \overline{y^{2}}\right)^{-\frac{1}{2}} \exp \left(-y^{2} / 2 \overline{y^{2}}\right), \quad \overline{\left(y^{2}\right.}=\left(\frac{2}{3} \alpha t\right)^{3}\right),
$$

where the variable $q$ is the ensemble-average value taken over concentration distributions arising from the release of a large number of identical clouds of marked fluid.

The forms of the two solutions, (5) and (7), are significantly different (see table 1 and figure 4). The Richardson curve has twice the modal value, four times the value of the second moment and approximately eighty times the value of the 
fourth moment of the Batchelor curve. These large differences allow a test of the two hypotheses, using relatively crude observations.

\section{Experimental procedure}

At a site $1 \frac{1}{2}$ miles offshore at Bai du Dore on Lake Huron, continuous plumes were formed by injecting a neutrally buoyant solution of Rhodamine B, methyl alcohol and water into the lake surface using a constant head syphon. The local bottom topography was smooth and the water depth approximately $30 \mathrm{~m}$. The ratio $h$, of the water depth to the distance to shore was much smaller than the

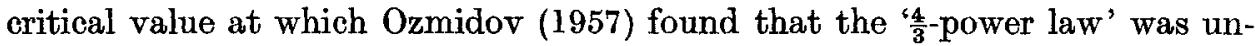
representative. $\uparrow$

$\begin{array}{ccc} & \text { Batchelor } & \text { Richardson } \\ \int_{-\infty}^{\infty} q(y, t) d y & 1 & 1 \\ q(0, t) & \left((2 \pi)^{\frac{1}{3}}\left(\frac{2}{3} \alpha t\right)^{\frac{3}{2}}\right)^{-1} & 3\left((6 \pi)^{\frac{1}{3}}\left(\frac{2}{3} \alpha t\right)^{\frac{3}{2}}\right)^{-1} \\ \overline{y^{2}} & \left(\frac{2}{3} \alpha t\right)^{3} & \frac{35}{9}\left(\frac{2}{3} \alpha t\right)^{3} \\ \overline{y^{4}} & 3\left(\frac{2}{3} \alpha t\right)^{6} & (5005 / 27)\left(\frac{2}{3} \alpha t\right)^{6} \\ \overline{y^{4}} /\left(\overline{y^{2}}\right)^{2} & 3 & 429 / 35\end{array}$

TABLE 1. A comparison of the Batchelor and Richardson forms of distance-neighbour functions

Plume-sampling stations were marked with buoyed flag standards whose positions were recorded from onshore theodolite stations. A catamaran motor boat, to which two submersible pumps were suspended at depths of 1 and $2 \mathrm{~m}$ below the lake surface, was used to cross the plume in the line of the flags. The vessel speed was approximately matched with the pump intake velocity (about $2 \mathrm{~m} \mathrm{sec}^{-1}$ ). Fluid from the pump intakes was passed through two Turner opendoor fluorometers which produced a recorded electrical signal proportional to the sample fluorescence. A linear relationship was found between this measure of fluorescence and the concentration of Rhodamine B dye mixture. As many as 25 crossings were made at one plume sampling station.

Measurements of wind speeds, water-temperature profiles and near-surface currents were made during the experiments, further details of which are given by Sullivan (1965).

\section{Experimental results}

Over a period of 5 days of making measurements a total of nine plume-sampling stations were used to collect 209 records of the concentration distribution across the plumes. Samples were taken over periods of approximately $5 \mathrm{~h}$ during which

$\dagger$ Ozmidov found, for values of $h>0 \cdot 1$, that the mean-square separation rate for floating. objects is described by $\frac{1}{2} d y^{2} / d t=\gamma y^{n}$,

where $\gamma$ and $n$ are functions of $h$. 
the conditions near the lake surface were nearly steady, and a well-defined thermocline structure was present. The presence of the thermocline, at depths ranging from 5 to $14 \mathrm{~m}$, limited the vertical diffusion and the dye plume appeared to become uniformly spread within the region bounded by the free surface and the top of the thermocline. It was also assumed that the longitudinal variation

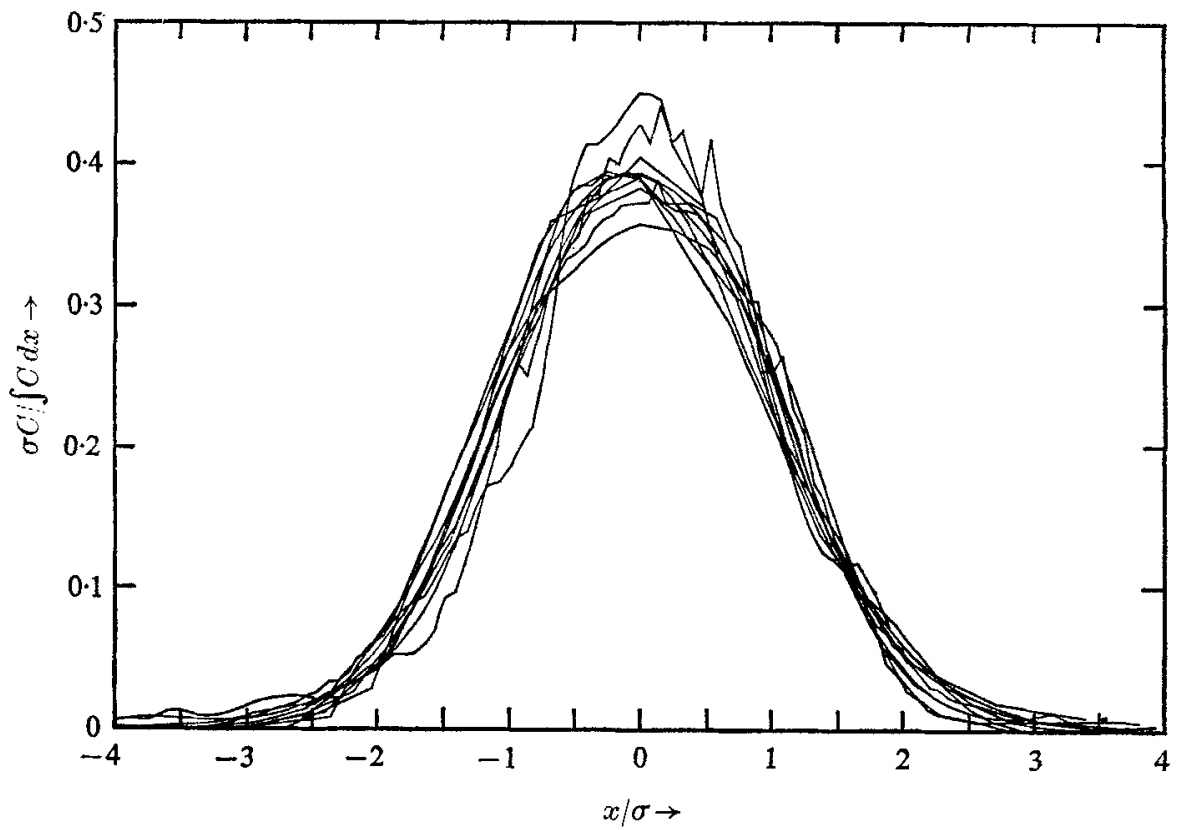

Figure 1. Section average concentration curves.

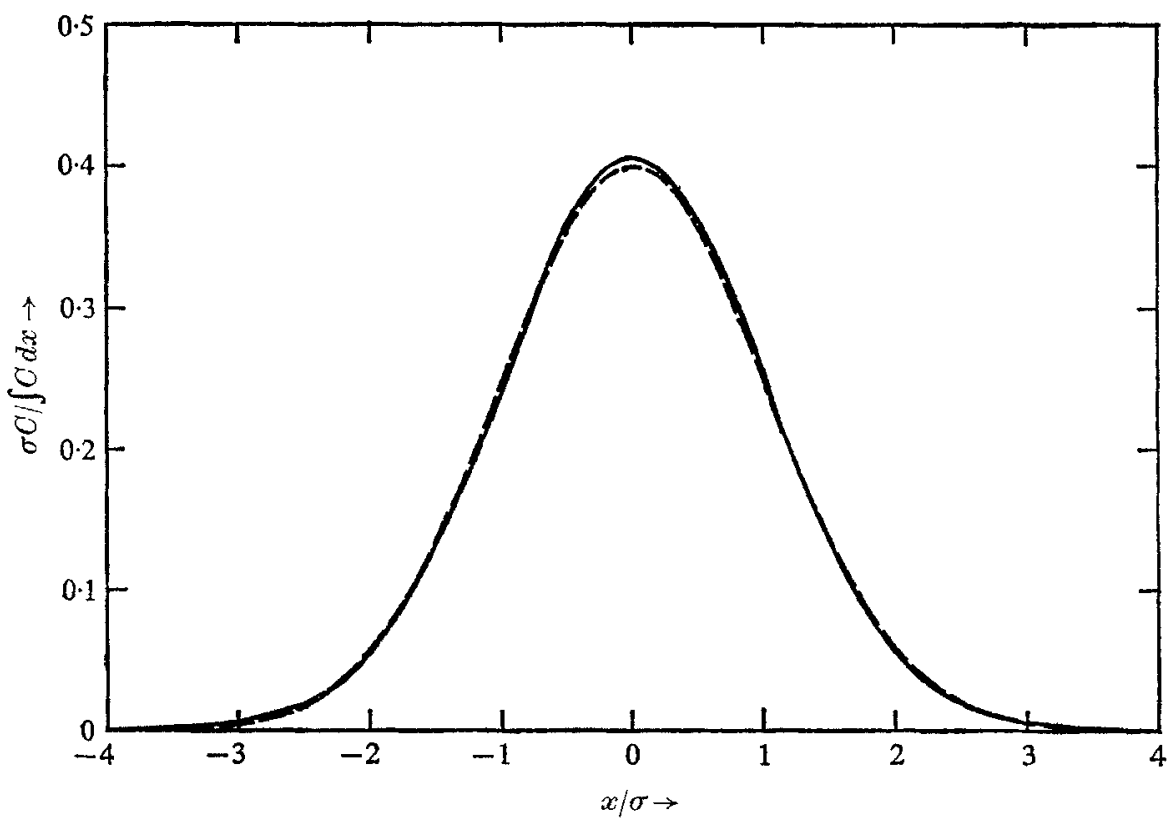

Figure 2. Average concontration compared with Gaussian. 
of the concentration was slight so that longitudinal mixing could be ignored and the experimental results thus compared with predictions based on a onedimensional model.

The average concentration curve for each sampling station, found by aligning the centre of gravity of individual records, was very nearly Gaussian (see figure 1). The average kurtosis value was $3 \cdot 2$, compared with the Gaussian value of 3 . In figure 2 , the average value of the 209 individual concentration curves is compared with the Gaussian curve. The average concentration curves found in this way have been discussed by Csanady (1966) in the context of dispersal predictions and pollution control.

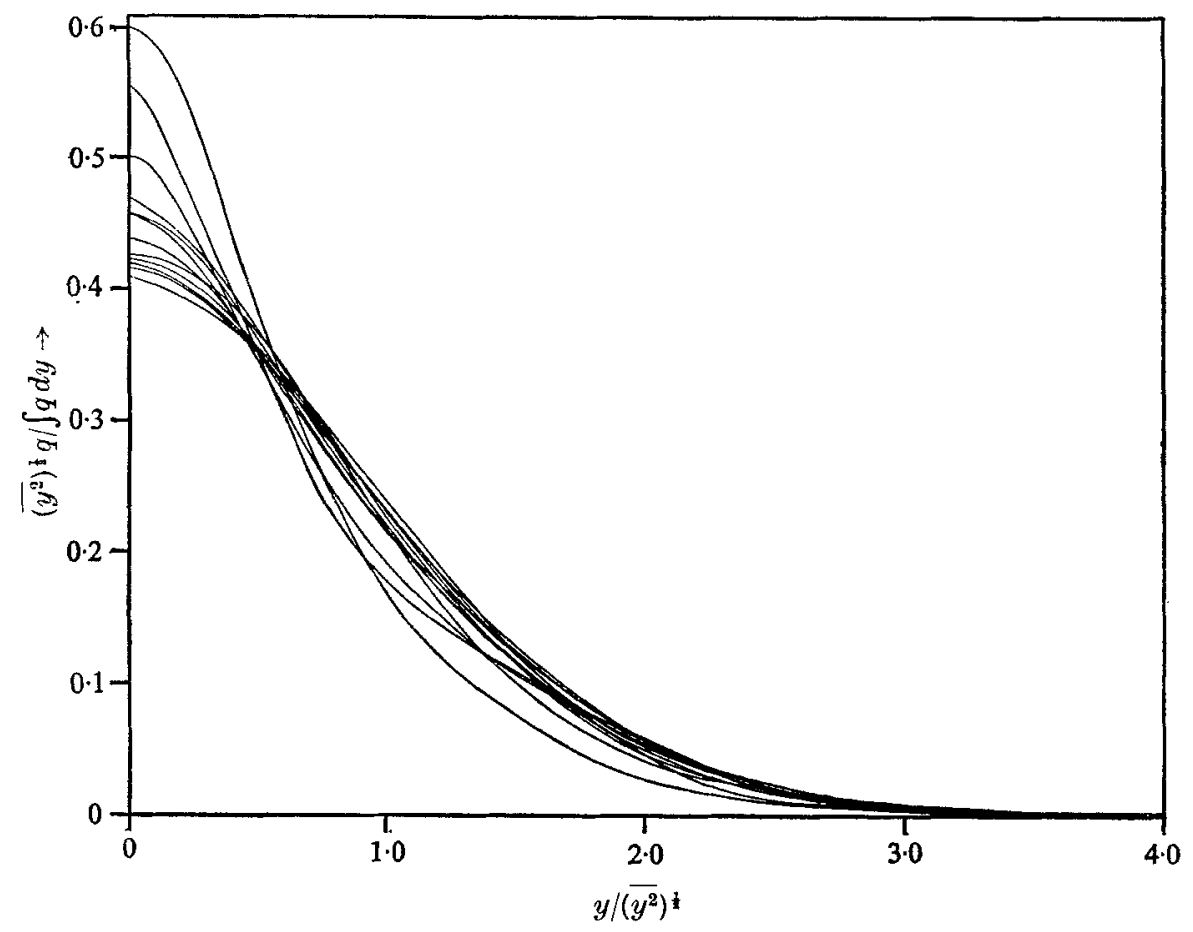

Figure 3. Average distance-neighbour function at each sample station.

A distance-neighbour function was formed for each concentration record and the average result for each sampling station is shown in figure 3. The variation about the mean curve at each sampling station was as large as the variation. between the stations (cf. figure 3 ); the average results, at a given station, are in reasonable agreement with the Gaussian form predicted by Batchelor. Using all the 209 measurements of the distance-neighbour function a mean curve has been compiled and is shown in figure 4 together with the theoretical curves of Batchelor (1952) and Richardson (1926). The curve derived from the experiments appears to be slightly leptokurtic with respect to the Gaussian form. This is believed to be a result of the influence of the high wave-number fluctuations in the concentration curves on the measured distance-neighbour function at small $y$. This suggests that the flow Reynolds numbers available for the experiments were 


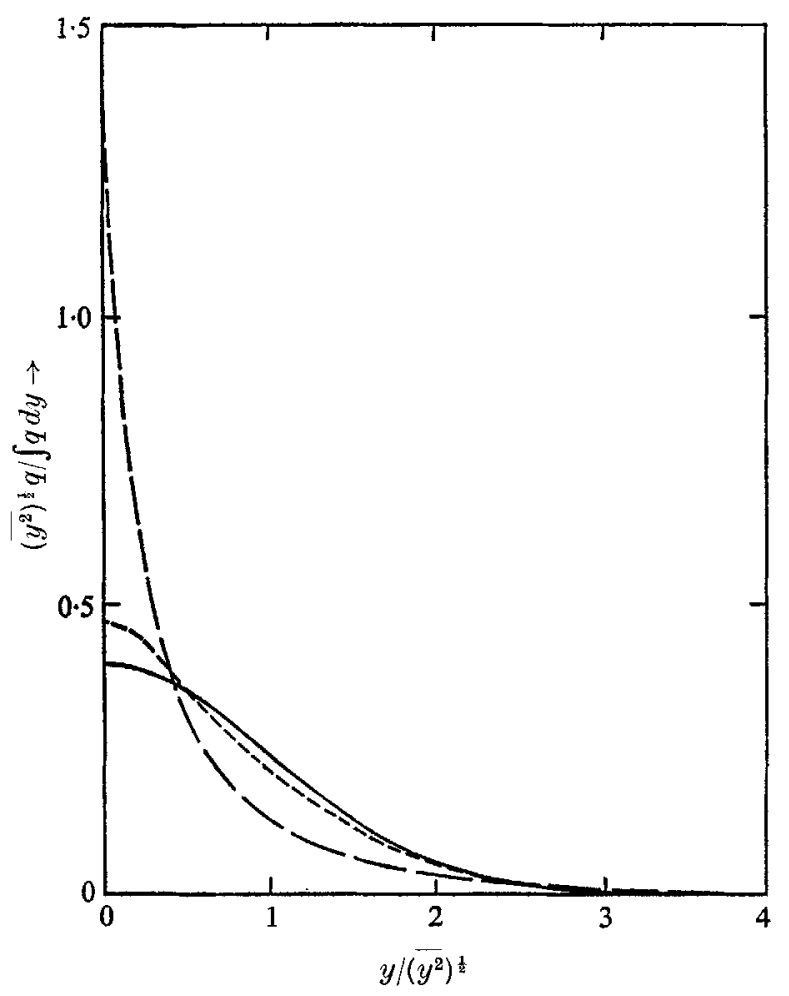

Figure 4. Average distance-neighbour function from 209 realizations of $C$ compared with suggestions of Batchelor and Richardson. __, Batchelor;_-_-, empirical;_- -, Richardson.

$\begin{array}{ccccccc}\text { Expt. } & \begin{array}{c}\text { Sect. } \\ \text { no. }\end{array} & \begin{array}{c}\text { Sample } \\ \text { distance } \\ (\mathrm{m})\end{array} & \begin{array}{c}\text { Average } \\ \text { velocity } \\ (\mathrm{cm} / \mathrm{sec})\end{array} & \begin{array}{c}\text { Thermocline } \\ \text { depth } \\ (\mathrm{m})\end{array} & \begin{array}{c}\text { Number } \\ \text { of } \\ \text { crossings }\end{array} & \alpha \\ 1 & 1 & 238 & 12 \cdot 7 & \mathbf{1 3 \cdot 7} & 25 & 0 \cdot 133 \\ 1 & 2 & 488 & 12 \cdot 7 & 10 \cdot 5 & 8 & 0 \cdot 090 \\ 2 & 1 & 146 & 14 \cdot 0 & 8 & 25 & 0 \cdot 080 \\ 2 & 2 & 390 & 14 \cdot 0 & 8 & 20 & 0 \cdot 121 \\ 3 & 1 u & 193 & 17 \cdot 1 & 7 & 26 & 0 \cdot 139 \\ & l & - & - & - & 27 & 0 \cdot 091 \\ 3 & 2 u & 366 & 17 \cdot 1 & 7 & 16 & 0 \cdot 089 \\ & l & - & - & - & 16 & 0 \cdot 070 \\ 4 & 1 u & 183 & 17 \cdot 7 & 5 \cdot 3 & 12 & 0 \cdot 193 \\ & l & - & - & - & 9 & 0 \cdot 122 \\ 4 & 2 & 329 & 17 \cdot 7 & 6 & 10 & 0 \cdot 166\end{array}$

$u, l$ refer to upper and lower pump readings.

Thermocline depth is defined as depth in metres at which the temperature is one degree $\mathrm{C}$ below that of the stirred surface layer.

TABLE 2 
low; that is, at higher Reynolds number flows a larger proportion of the turbulent energy would appear at wave-numbers that contribute most effectively in dispersing the high wave-number concentration fluctuations. As the plumes become more dispersed further downstream, the comparison between the measured distance-neighbour function and the form predicated by Batchelor may be expected to improve.

Equation (7) was used to calculate experimental values for $\alpha$ (see table 2). The average value of $\alpha=0.12$ is between the value of $\alpha=0.2$ suggested by Richardson for the atmosphere, and the values 0.07 and 0.01 found respectively by Richardson \& Stommel (1948) and by Ozmidov (1961) for bodies floating on the sea surface. The experimental average rate of energy dissipation using (2) (i.e. $\epsilon=\alpha^{3} / c^{3}$ ) is $2 \cdot 1 \times 10^{-3} \mathrm{~cm}^{2} \mathrm{sec}^{-3}$ for the value of $c=1 \cdot 0$. This is at the low end of the range of estimates $\left(1 \cdot 7 \times 10^{-3}<\epsilon<8 \times 10^{-2}\right)$ by authors using various methods and summarized by Ozmidov (1961). For data collected from objects floating on the sea surface Ozmidov has used $c=0 \cdot 1$ to calculate $\epsilon$.

The approximately normal distribution of $q$, found experimentally, is a reasonable result which according to Batchelor (1952) could be interpreted, from a loose application of the central limit theorem, as describing the probable separation of two typical marked fluid elements, following a large succession of almost independent, but on the average increasing, relative motions. The experimental data is crude by the standards of laboratory measurements but it seems sufficiently accurate to distinguish between the two suggested descriptions for the characteristics of a dispersing cloud of passively marked fluid.

The experimental program was carried out using the facilities of the Great Lakes Institute, University of Toronto, and received financial support by the Atomic Energy of Canada Limited and by the National Research Council of Canada through Grant A 1820. Recent analytical work has been supported by Grant 16070 DGY from the United States Federal Water Pollution Control Administration at the California Institute of Technology, Pasadena, California, U.S.A.

\section{REFERENCES}

BATCHeLOR, G. K. 1950 Quart. J. Roy. Met. Soc. 76, 133-146.

Batchelor, G. K. 1952 Proc. Camb. Phil. Soc. 48, 345-362.

Csanady, G. T. 1966 Proceedings of Conference on Great Lakes Research, 9th Ann Arbor, (Great Lakes Research Division Publication no. 15), 283-293.

Ozmıdov, R. V. 1957 Akademiia Nauk SSSR, Bulletin Geophysics Series, no. 6, 73-83.

Ozmmov, R. V. 1960 Akademiia Nauk SSSR, Doklady Earth Sciences, Sections 126, $536-538$.

Ozmidov, R. V. 1961 Akademiia Nauk SSSR, Bulletin Geophysics Series, no. 8, 821-823.

Richardoson, L. F. 1926 Proc. Roy. Soc. A 110, 709-737.

Richardson, L. F. 1929 Beitr. Phys. frei Atmos. 15, 24-29.

Richardson, L. F. \& Stommed, H. $1948 J$. Meteorol. 5, 238-240.

Sullivan, P. J. 1965 M.Sc. Thesis, University of Waterloo. 